\title{
HYPERELLIPTIC GRAPHS AND METRIZED COMPLEXES
}

\author{
YOAV LEN \\ Department of Combinatorics \& Optimization, University of Waterloo, \\ 200 University Avenue West, Waterloo, Ontario, Canada; \\ email: yoav.len@uwaterloo.ca
}

Received 27 June 2016; accepted 26 April 2017

\begin{abstract}
We prove a version of Clifford's theorem for metrized complexes. Namely, a metrized complex that carries a divisor of degree $2 r$ and rank $r$ (for $0<r<g-1$ ) also carries a divisor of degree 2 and rank 1 . We provide a structure theorem for hyperelliptic metrized complexes, and use it to classify divisors of degree bounded by the genus. We discuss a tropical version of Martens' theorem for metric graphs.
\end{abstract}

2010 Mathematics Subject Classification: 14C20, 14H51, 14H40, 14T05

\section{Introduction}

For a smooth algebraic curve, Clifford's theorem states that a divisor of rank $r$ has degree at least $2 r$, and when $0<r<g-1$, equality may only be obtained for hyperelliptic curves $[4, \mathrm{Ch}$. III]. The first part of the theorem follows immediately from Riemann-Roch, whereas the second part requires more subtle geometric methods.

With the development of tropical and non-Archimedean geometry in recent years, it was observed that many theorems from classical algebraic geometry have combinatorial analogs in tropical geometry. Baker and Norine introduced divisors on finite graphs, and showed that they satisfy a Riemann-Roch theorem [8]. Their results were later generalized by various authors to metric and weighted graphs [3, 11, 20]. Via Baker's Specialization Lemma [5], such results provide new combinatorial techniques for studying algebraic curves.

(c) The Author 2017. This is an Open Access article, distributed under the terms of the Creative Commons Attribution licence (http://creativecommons.org/licenses/by/4.0/), which permits unrestricted re-use, distribution, and reproduction in any medium, provided the original work is properly cited. 
Subsequently, Amini and Baker introduced metrized complexes, a common generalization of metric graphs and algebraic curves, and prove a Riemann-Roch theorem in that setting [1, Theorem 1.3]. While these objects tend to be more involved than graphs, they also capture more algebraic information, and provide a much stronger tool in some cases. For instance, Katz, Rabinoff and Zurich-Brown apply the first part of Clifford's theorem for the metrized complex associated to a semistable model of a curve, to bound the number of its rational points, thus proving a weak version of the Bombieri-Lang conjecture [14].

Similarly to the algebraic case, an analog of the first part of Clifford's theorem follows immediately from Riemann-Roch. However, the methods used to prove the second part do not carry well into the tropical world. Nevertheless, it is shown in [10] that the full extent of Clifford's theorem holds for metric graphs. Our main result is an extension of the theorem to metrized complexes.

THEOREM (3.4). Let $\mathfrak{C}$ be a metrized complex of genus $g$, and suppose that for some $0<r<g-1$ there is a divisor class $\delta$ of degree $2 r$ and rank $r$. Then $\mathfrak{C}$ is hyperelliptic.

By hyperelliptic we mean a metrized complex having a divisor of degree 2 and rank 1. For a smooth curve, the existence of such a divisor induces a double cover of a line. Similarly, for a metric graph, such a divisor implies the existence of a harmonic map of degree 2 to a tree [9, Theorem 1.3]. The analogous statement for metrized complexes is no longer true, as shown in [2, Example 4.14] and [1, Remark 5.13]. Nevertheless, just as hyperelliptic graphs can be pictured as two isomorphic trees meeting at their leaves [9, Theorem 1.3], we show that hyperelliptic metrized complexes consist of two isomorphic trees, meeting along hyperelliptic algebraic curves. See Lemma 3.5 for a precise statement. This description allows us to classify all the effective divisors whose degree is bounded by the genus.

THEOREM (3.6). Let $\mathfrak{C}$ be a hyperelliptic metrized complex. Then every divisor class $\delta$ of degree $d$ and rank $r$ with $0 \leqslant r \leqslant d \leqslant g$ is of the form $r \cdot \mathfrak{g}_{2}^{1}+p_{2 r+1}+$ $\cdots+p_{d}$.

In the appendix, we discuss a possible tropical version of Martens' theorem, which is a refinement of Clifford's theorem. The results presented there are joint work with David Jensen.

Our strategy for proving the main theorem is partly inspired by the techniques used in [10]. However, our argument is entirely self-contained. In particular, it provides an independent proof of Clifford's theorem for graphs by considering 


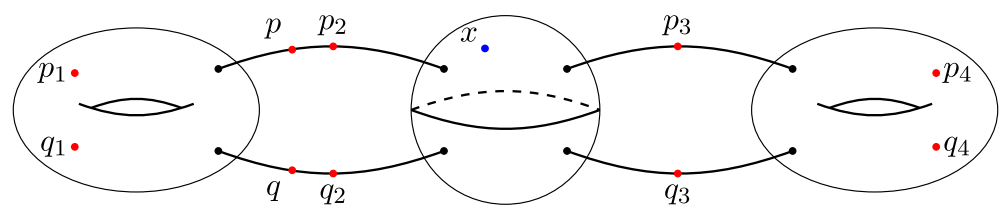

Figure 1. The metrized complex $\mathfrak{C}$.

metrized complexes in which all the components are rational. Before delving into the proof and introducing various notations, we begin with an example to demonstrate the strategy.

EXAMPLE 1.1. Let $\mathfrak{C}$ be the metrized complex of genus 4, depicted in Figure 1 (see Section 2 for notations regarding metrized complexes). All its edges have the same length, the points $p_{3}, q_{3}, p_{4}, q_{4}$ are in the middle of the edges, and $p, q$ are of equal distance from $p_{2}, q_{2}$, respectively. Suppose that $p_{1}+q_{1}$ and $p_{4}+q_{4}$ are equivalent to the pair of nodes corresponding to the incoming edges at their respective components. The points $p, p_{1}, p_{2}, p_{3}, p_{4}$ form a rank determining set for $\mathfrak{C}$, so to show that a divisor class has rank 1 , it suffices to show that the union of its different representatives contains each of them.

The divisor $\mathcal{D}=4 \cdot(x)$ has rank 2 , and therefore, has a representative $\mathcal{D}^{\prime}$ containing $p_{1}+p_{2}$. It is straightforward to check that this representative is exactly $p_{1}+p_{2}+q_{1}+q_{2}$. Similarly, it has a representative containing $p_{2}+p_{3}$, namely, $p_{2}+p_{3}+q_{2}+q_{3}$. It follows that

$$
p_{1}+p_{2}+q_{1}+q_{2} \simeq p_{2}+p_{3}+q_{2}+q_{3},
$$

so by linearity, $p_{1}+q_{1} \simeq p_{3}+q_{3}$. By repeating this process for all the different combinations $p_{i}+p_{j}$ and $p_{j}+p_{k}$, we see that

$$
p_{1}+q_{1} \simeq p_{2}+q_{2} \simeq p+q \simeq p_{3}+q_{3} \simeq p_{4}+q_{4} .
$$

Therefore, $p_{1}+q_{1}$ has rank 1 , and $\mathfrak{C}$ is hyperelliptic.

In the next section, we show that every metrized complex of genus $g$ which satisfies the conditions of the main theorem contains a divisor of degree $2 g$, with similar properties to the divisor $\sum p_{i}+\sum q_{i}$ in the example above.

\section{Preliminaries}

In what follows, we assume familiarity with the theory of tropical divisors and metrized complexes. We refer the reader to [7] for an extensive exposition on the 
topic, and to [1] for a more thorough treatment. Roughly speaking, a metrized complex is a generalization of a metric graph, obtained by placing smooth curves at the vertices, and defining linear equivalence in a way that combines chip firing on the graph and linear equivalence on the curves.

DEFINITION 2.1. A metrized complex is hyperelliptic if it has a divisor of degree 2 and rank 1.

We refer to the algebraic curves placed at the vertices as components. For each vertex $v$, denote $C_{v}$ the corresponding component and $g_{v}$ its genus. The point of a component $C_{v}$ associated to an edge is referred to as a node. The metric graph $\Gamma$, obtained by removing the components is called the underlying graph of $\mathfrak{C}$. There is a natural map which takes divisors on $\mathfrak{C}$ to divisors on $\Gamma$. By abuse of notation, we often identify a divisor on $\mathfrak{C}$ with its image without mention. The elements of a divisor are referred to as chips. For an algebraic curve or a metric graph we denote by $W_{d}^{r}$ the set of its divisor classes of degree $d$ and at least rank $r$, or simply $W_{d}$ when $r=0$.

DEFINITION 2.2. A divisor on a graph or a metrized complex is said to be rigid if it is the unique effective divisor in its class.

On an algebraic curve, a divisor is rigid if and only if it is effective and has rank zero. On metric graphs, rank zero is a necessary but not sufficient condition for rigidness. However, as seen by the following lemma, rigid divisors are ubiquitous.

LEMMA 2.3. Let $\Gamma$ be a metric graph of genus $h>0$, and let $K$ be its canonical divisor. Then there is a divisor $P$ of degree $h-1$ such that $P$ and $K-P$ are rigid. Moreover, there is an open set of such divisors in the space $W_{h-1}(\Gamma)$ of effective divisor classes of degree $h-1$.

Proof. The space $W_{h-1}(\Gamma)$ is the Minkowski sum of $h-1$ copies of $W_{1}(\Gamma)$ in the Jacobian of $\Gamma$. Since the latter is the image of $\Gamma$ under the Abel-Jacobi map, the former is a connected polyhedral complex of pure dimension $h-1$.

Let $D$ be a nonrigid effective divisor of degree $h-1$. We begin by showing that $D$ has a representative with at least one chip at a vertex. Suppose that $D$ is supported on the interior of edges of $\Gamma$. Since $D$ is nonrigid, removing its support disconnects the graph [13, Theorem 13]. Let $p_{1}, \ldots, p_{k}$ be a minimal subset of the support that disconnects the graph, and let $e_{1}, \ldots, e_{k}$ be the edges that contain these points respectively. Then $\Gamma \backslash\left\{e_{1}, \ldots, e_{k}\right\}$ is a disjoint union of two graphs $\Gamma_{1}$ and $\Gamma_{2}$. Then we can 'move' $p_{1}, \ldots, p_{k}$ together until they reach $\Gamma_{1}$ : Let $d$ be 
the minimal distance from $\Gamma_{1}$ to $p_{1}, \ldots, p_{k}$. Define a piecewise linear function $f$ whose value on $\Gamma_{1}$ is 0 , has slope -1 on the first segment of length $d$ on each edge $e_{i}$ emanating from $\Gamma_{1}$, and is constant on the rest of the graph. Then $D+\operatorname{div}(f)$ is effective with a representative on a vertex.

The set of divisor classes with a chip on a vertex has dimension strictly smaller than $h-1$. Therefore, there is a dense open set in $W_{h-1}(\Gamma)$ classifying rigid divisors. Since the map taking a divisor $P$ to $K-P$ is a linear bijection between $W_{h-1}$ to itself, there is an open dense set for which both $P$ and $K-P$ are rigid.

The following lemma is a useful tool for dealing with the graph and algebraic parts of divisors separately (cf. [18, Theorem 4.3] and [1, Proposition 2.1]).

LEMMA 2.4. Let $\mathcal{D}$ be a divisor of rank $r>0$, let $r=r_{0}+\sum_{v \in V(\Gamma)} r_{v}$ be a partition of $r$, and let $E$ be an effective divisor of degree $r_{0}$ on $\Gamma$. Then $\mathcal{D}$ is equivalent to an effective divisor that contains $E$, whose restriction to each component of $\mathfrak{C}$ has rank $r_{v}$.

Proof. For each rational function $\mathfrak{f}$ on $\mathfrak{C}$ such that $\mathcal{D}+\mathfrak{f}$ is effective and contains $E$, let $s_{f}$ be the collection of its incoming slopes at the components of $\mathfrak{C}$. Let $S$ be the set of all such $s_{f}$. For $s \in S$ and a component $C_{v}$, let

$$
T_{s, v}=\left\{D \in W_{r_{v}}\left(C_{v}\right) \mid \text { there exists } \mathfrak{f} \text { with } s_{\mathfrak{f}}=s \text { such that } D \leqslant \mathcal{D}+\operatorname{div}(\mathfrak{f})\right\} .
$$

The restriction to $C_{v}$ of divisors of the form $\mathcal{D}+\operatorname{div}(\mathfrak{f})$ for $s_{\mathfrak{f}}=s$ are all equivalent to each other, and $T_{s, v}$ is the entire space $W_{r_{v}}\left(C_{v}\right)$ if and only those restrictions form a divisor class of rank $r_{v}$.

Let $T_{s}=\prod_{v \in V(\Gamma)} T_{s, v}$. Since $\mathcal{D}$ has rank $r$, the union of all the sets $T_{s}$ is $\prod_{v \in V(\Gamma)} W_{r_{v}}\left(C_{v}\right)$. Furthermore, since each $T_{s}$ is closed, and $S$ is finite, there must be $s \in S$ such that whenever $s_{\mathfrak{f}}=s$, the divisor $\mathcal{D}+\operatorname{div}(\mathfrak{f})$ has rank $r_{v}$ at every component $C_{v}$.

For the rest of the section, we fix a metrized complex $\mathfrak{C}$ of genus $g>1$, whose underlying graph $\Gamma$ has genus $h$. Denote by $\mathcal{K}$ the canonical class of $\mathfrak{C}$.

Proposition 2.5. Suppose that $h \geqslant 2$. Then there exist effective divisors $\mathcal{P}, \mathcal{Q}$ of degree $g-1$ such that:

(1) $\mathcal{P}+\mathcal{Q} \simeq \mathcal{K}$.

(2) $\mathcal{P}, \mathcal{Q}$ are rigid.

(3) For each component $C_{v}$ the restriction of $\mathcal{P}, \mathcal{Q}$ to $C_{v}$ is rigid and does not meet the nodes. 
Moreover, there is an open set $\sigma$ classifying such divisors in $\prod_{v \in V(\Gamma)} W_{g_{v}}\left(C_{v}\right) \times$ $W_{h-1}(\Gamma)$.

Proof. Fix rigid divisors $P+Q \simeq K$ on $\Gamma$ as in Lemma 2.3. By Lemma 2.4, there is a representative $\mathcal{K}^{\prime}$ of $\mathcal{K}$ that contains $P$, whose restriction to each component $C_{v}$ has rank $g_{v}$. Riemann-Roch for curves implies that this restriction has degree at least $2 g_{v}$. By subtracting $2 g_{v}$ chips from each component, and forgetting the metrized complex structure, we obtain a divisor which is equivalent to the canonical divisor $K$ of $\Gamma$, and contains $P$. By Lemma 2.3, it is precisely the divisor $P+Q$. Therefore, the restriction of $\mathcal{K}^{\prime}$ to each component has exactly $2 g_{v}$ chips. Since the set of rigid divisors of degree $g_{v}$ on $C_{v}$ is open and dense in $W_{g_{v}}\left(C_{v}\right)$, and the divisors $P, Q$ vary in an open dense set of $W_{h-1}(\Gamma)$, there is an open dense set in the product satisfying the desired properties.

Proposition 2.6. Suppose that $h$ is either 0 or 1 . Then there exist effective divisors $\mathcal{P}, \mathcal{Q}$ of degree $g-1$ such that:

(1) $\mathcal{P}+\mathcal{Q} \simeq \mathcal{K}$.

(2) $\mathcal{P}, \mathcal{Q}$ are rigid.

(3) For each component $C_{v}$ the restriction of $\mathcal{P}, \mathcal{Q}$ to $C_{v}$ is rigid and does not meet the nodes.

Moreover, there is an open set $\sigma$ classifying such divisors in $W_{g_{v_{0}}-1}\left(C_{v_{0}}\right) \times$ $\prod_{v_{0} \neq v \in V(\Gamma)} W_{g_{v}}\left(C_{v}\right) \times W_{h}(\Gamma)$ for some vertex $v_{0}$.

Proof. Choose a vertex $v_{0}$ with $g_{v_{0}}>0$, which exists since we assumed that $g>1$. By Lemma 2.4, together with Riemann-Roch for curves, the divisor $\mathcal{K}$ is equivalent to a divisor whose restriction to $C_{v_{0}}$ has degree $2 g_{v_{0}}-2$ and rank $g_{v_{0}}-1$, its restriction to any other component $C_{v}$ has degree $2 g_{v}$ and rank $g_{v}$, and its restriction to the edges of the underlying graph $\Gamma$ has $2 h$ chips. Moreover, the restriction to each component can be chosen so that it consists of a pair of rigid divisors. Now choose $\mathcal{P}$ to consist of the first summands of those pairs and $h$ of the graph points, and $\mathcal{Q}$ to consist of the second summands.

Recall that a set $\mathcal{R}$ is said to be rank determining if it suffices to consider points of $\mathcal{R}$ when computing the ranks of divisors. More precisely, $\mathcal{R}$ is rank determining if the rank of every divisor $\mathcal{D}$ is the largest number $r$ such that $\mathcal{D}-p_{1}-\cdots-p_{r}$ is equivalent to an effective divisor, for every choice of $p_{1}, \ldots, p_{r}$ in $\mathcal{R}$. The following lemma is a mild generalization of [1, Theorem A.1]. We leave it for the reader to make the necessary changes. 
LEMMA 2.7. Let $\mathcal{R}$ be a divisor of degree $g+1$ with the following properties:

(1) $\mathcal{R}$ has $h+1$ graph points (where $h$ is the genus of $\Gamma$ ), and the graph obtained from $\Gamma$ by removing $h$ of them is a tree.

(2) The restriction of $\mathcal{R}$ to every component $C_{v}$ is a rigid divisor of degree $g_{v}$.

Then $\mathcal{R}$ is a rank determining set.

\section{Hyperelliptic metrized complexes}

3.1. Clifford's theorem. In this section, we assume the existence of a divisor class $\delta$ of degree $2 r$ and rank $r$ for some $1<r<g-1$, and conclude that $\mathfrak{C}$ is hyperelliptic. Let $\sigma$ be the open set of rigid divisors constructed in Propositions 2.5, 2.6. By construction, for each $\mathcal{P}^{\prime} \in \sigma$, there is a unique $\mathcal{Q}^{\prime} \in \sigma$ such that $\mathcal{P}^{\prime}+\mathcal{Q}^{\prime} \simeq \mathcal{K}$. Let $\mu: \sigma \rightarrow \sigma$ be the map which assigns $\mathcal{Q}^{\prime}$ to $\mathcal{P}^{\prime}$. Fix a pair $\mathcal{P} \in \sigma$ and $\mathcal{Q}=\mu(\mathcal{P})$.

DEFInition 3.1. For divisors $\mathcal{D}, \mathcal{E}$, denote

$$
(\mathcal{D} \cap \mathcal{E})(v)=\min (\mathcal{D}(v), \mathcal{E}(v))
$$

and

$$
(\mathcal{D} \cup \mathcal{E})(v)=\max (\mathcal{D}(v), \mathcal{E}(v)) .
$$

For a divisor $\mathcal{D}$, we define its $\mathcal{P}$-part as $\mathcal{D}^{\mathcal{P}}=\mathcal{D} \cap \mathcal{P}$, and its $\mathcal{Q}$-part as $\mathcal{D}^{\mathcal{Q}}=$ $\mathcal{D} \cap \mathcal{Q}$.

LEMMA 3.2. If $\mathcal{D} \in \delta$ is effective and $\operatorname{deg}\left(\mathcal{D}^{\mathcal{P}}\right)=r$, then $\mathcal{D}$ is supported on $\mathcal{P}+\mathcal{Q}$. In particular, $\mathcal{D}=\mathcal{D}^{\mathcal{P}}+\mathcal{D}^{\mathcal{Q}}$.

Proof. By Riemann-Roch, the divisor $\mathcal{K}-\mathcal{D}$ has rank $g-1-r$. Let $\mathcal{E}$ be an effective divisor that contains $\mathcal{P}-\mathcal{D}^{\mathcal{P}}$ and is equivalent to $\mathcal{K}-\mathcal{D}$. Then $\mathcal{D}+\mathcal{E}$ is canonical, and as such, equivalent to $\mathcal{P}+\mathcal{Q}$. Therefore, $\mathcal{D}+\mathcal{E}-\mathcal{P} \simeq \mathcal{Q}$. But $\mathcal{D}+\mathcal{E}-\mathcal{P}$ is effective and $\mathcal{Q}$ is rigid, so $\mathcal{D}+\mathcal{E}-\mathcal{P}$ is exactly $\mathcal{Q}$. By adding $\mathcal{P}$ to both sides, $\mathcal{D}+\mathcal{E}=\mathcal{P}+\mathcal{Q}$.

By now, we know that there is a correspondence between the subsets of size $r$ of $\mathcal{P}$ and $\mathcal{Q}$. Next, we show that the correspondence respects unions and intersections. 
Proposition 3.3. If $\mathcal{D}_{1}, \ldots, \mathcal{D}_{k}$ are effective representatives of $\delta$ such that $\operatorname{deg}\left(\mathcal{D}_{i}^{\mathcal{P}}\right)=$ for each $i$, then

$$
\operatorname{deg}\left(\mathcal{D}_{1}^{\mathcal{P}} \cap \cdots \cap \mathcal{D}_{k}^{\mathcal{P}}\right)=\operatorname{deg}\left(\mathcal{D}_{1}^{\mathcal{Q}} \cap \cdots \cap \mathcal{D}_{k}^{\mathcal{Q}}\right)
$$

and

$$
\operatorname{deg}\left(\mathcal{D}_{1}^{\mathcal{P}} \cup \cdots \cup \mathcal{D}_{k}^{\mathcal{P}}\right)=\operatorname{deg}\left(\mathcal{D}_{1}^{\mathcal{Q}} \cup \cdots \cup \mathcal{D}_{k}^{\mathcal{Q}}\right)
$$

Proof. For each $i=1, \ldots, k$, let $\sigma_{i}$ be the subset of $\sigma$ consisting of divisors that contain $\mathcal{P}-\mathcal{D}_{i}^{\mathcal{P}}$. Similarly, let $\tau_{i}$ be the subset of $\sigma$ of divisors containing $\mathcal{Q}-\mathcal{D}_{i}^{\mathcal{Q}}$. We claim that the image of $\left.\mu\right|_{\sigma_{i}}$ is contained in $\tau_{i}$ for each $i$. Indeed, let $\mathcal{P}_{i} \in \sigma_{i}$, and let $\mathcal{E}_{i}=\mathcal{P}_{i}-\mathcal{P}+\mathcal{D}_{i}^{\mathcal{P}}$. By definition of $\sigma_{i}$, the divisor $\mathcal{P}_{i}$ contains $\mathcal{P}-\mathcal{D}_{i}^{\mathcal{P}}$, so $\mathcal{E}_{i}$ is effective (in fact, $\mathcal{E}_{i}$ can be thought of as a divisor obtained by perturbing $\left.\mathcal{D}_{i}^{\mathcal{P}}\right)$. Since $\operatorname{deg}\left(\mathcal{E}_{i}\right)=r$, there is a representative $\mathcal{D}_{i}^{\prime}$ of $\delta$ which contains $\mathcal{E}_{i}$. Now, $\mathcal{P}+\mathcal{Q}+\mathcal{D}_{i}^{\prime}-\mathcal{D}_{i}$ is effective because $\mathcal{D}_{i}$ is contained in $\mathcal{P}+\mathcal{Q}$, it is equivalent to $\mathcal{K}$ and contains $\mathcal{P}_{i}$, so by the definition of $\mu$, it equals $\mathcal{P}_{i}+\mu\left(\mathcal{P}_{i}\right)$. From

$$
\begin{aligned}
\mathcal{P} & +\mathcal{Q}+\mathcal{D}_{i}^{\prime}-\mathcal{D}_{i} \\
& =\mathcal{P}-\mathcal{D}_{i}^{\mathcal{P}}=\mathcal{Q}-\mathcal{D}_{i}^{\mathcal{Q}}+\mathcal{D}_{i}^{\prime}=\mathcal{P}_{i}+\mathcal{E}_{i}+\mathcal{Q}-\mathcal{D}_{i}^{\mathcal{Q}}+\mathcal{D}_{i}^{\prime} \\
& =\mathcal{P}_{i}+\left[\left(\mathcal{D}_{i}^{\prime}-\mathcal{E}_{i}\right)+\left(\mathcal{Q}-\mathcal{D}_{i}^{\mathcal{Q}}\right)\right],
\end{aligned}
$$

we see that $\mu\left(\mathcal{P}_{i}\right)$ contains $\mathcal{Q}-\mathcal{D}_{i}^{\mathcal{Q}}$, and in particular, is in $\tau_{i}$. We conclude that the restriction of $\mu$ to $\sigma_{1} \cap \cdots \cap \sigma_{k}$ maps to $\tau_{1} \cap \cdots \cap \tau_{k}$. The map $\mu$ is a bijection, so there is a one to one correspondence between divisors in $\sigma$ containing $\mathcal{P}-\mathcal{D}_{1}^{\mathcal{P}} \cap \cdots \cap \mathcal{D}_{k}^{\mathcal{P}}$ and divisors containing $\mathcal{Q}-\mathcal{D}_{1}^{\mathcal{Q}} \cap \cdots \cap \mathcal{D}_{k}^{\mathcal{Q}}$. In particular, $\mathcal{D}_{1}^{\mathcal{P}} \cap \cdots \cap \mathcal{D}_{k}^{\mathcal{P}}$ and $\mathcal{D}_{1}^{\mathcal{Q}} \cap \cdots \cap \mathcal{D}_{k}^{\mathcal{Q}}$ must have the same degree.

By the inclusion-exclusion principle, the cardinality of a union can be expressed as an alternating sum of the cardinalities of different intersection, which proves the second part of the proposition.

We are finally in a position to prove our main theorem.

THEOREM 3.4. Let $\mathfrak{C}$ be a metrized complex of genus $g$, and suppose that for $0<r<g-1$ there is a divisor class $\delta$ of degree $2 r$ and rank $r$. Then $\mathfrak{C}$ is hyperelliptic.

Proof. For each $p$ in the support of $\mathcal{P}$, let

$$
S_{p}=\left\{\mathcal{D} \in \delta \mid \operatorname{deg}\left(\mathcal{D}^{P}\right)=r, p \notin \mathcal{D}\right\} .
$$

By Proposition 3.3, $\bigcup_{\mathcal{D} \in S_{p}} \mathcal{D}^{\mathcal{Q}}$ contains all but a single point of $\mathcal{Q}$, which we denote $\pi_{\mathcal{P}}(p)$. The assignment $\mathcal{D}^{\mathcal{P}} \rightarrow \mathcal{D}^{\mathcal{Q}}$ is a bijection between the subsets of 
size $r$ of $\mathcal{P}$ and $\mathcal{Q}$, so we can reverse the process and conclude that $\pi_{\mathcal{P}}(p) \neq$ $\pi_{\mathcal{P}}\left(p^{\prime}\right)$ whenever $p \neq p^{\prime}$. In particular, any divisor $\mathcal{D} \in \delta$ whose $\mathcal{P}$-part has degree $r$, contains $\pi_{\mathcal{P}}(p)$ if and only if it contains $p$. We claim that all the divisors $p+\pi_{\mathcal{P}}(p)$ for $p$ in the support of $\mathcal{P}$ are equivalent. Indeed, choose $\mathcal{D}_{p}$ and $\mathcal{D}_{p^{\prime}}$ such that $p+\pi_{\mathcal{P}}(p) \leqslant \mathcal{D}_{p}, p^{\prime}+\pi_{\mathcal{P}^{\prime}}\left(p^{\prime}\right) \leqslant \mathcal{D}_{p^{\prime}}$, and $\operatorname{deg}\left(\mathcal{D}_{p}^{\mathcal{P}} \cap \mathcal{D}_{p^{\prime}}^{\mathcal{P}}\right)=r-1$. By the discussion above, $\operatorname{deg}\left(\mathcal{D}_{p} \cap \mathcal{D}_{p^{\prime}}\right)=2 r-2$, so

$$
0 \simeq \mathcal{D}_{p}-\mathcal{D}_{p^{\prime}}=p+\pi_{\mathcal{P}}(p)-p^{\prime}-\pi_{\mathcal{P}}\left(p^{\prime}\right),
$$

and it follows that $p^{\prime}+\pi_{\mathcal{P}}\left(p^{\prime}\right) \simeq p+\pi_{\mathcal{P}}(p)$. To finish the proof, we need to be extend this assignment to a rank determining set.

We first handle the case $h \geqslant 2$. Let $p_{1}, \ldots, p_{h-1}$ be the graph points of $\mathcal{P}$. Since $\sigma$ is open, we can assume, perhaps after perturbing the points, that all the graph points of $\mathcal{P}$ are in the interior of different edges. Next, we find an additional point $p_{1}^{\prime}$ in the interior of an edge so that $p_{1}^{\prime}+p_{1}+\cdots+p_{h-1}$ is rigid: by [13, Theorem 13], a divisor on a graph that is supported on the interior of edges is rigid if and only if removing it does not disconnect the graph. In particular, every such rigid divisor can be completed to a rigid divisor of degree $h$ that is supported on the interior of edges. Now, since $\sigma$ is open, we can choose this $p_{1}^{\prime}$, possibly after another perturbation of the points, so that both $\mathcal{P}$ and $\mathcal{P}-p_{1}+p_{1}^{\prime}$ are in $\sigma$. Denote $\mathcal{P}^{\prime}=\mathcal{P}-p_{1}+p_{1}^{\prime}$. By construction, the assignments $\pi_{\mathcal{P}}$ and $\pi_{\mathcal{P}^{\prime}}$ coincide on $\mathcal{P} \cap \mathcal{P}^{\prime}$. In particular, we have an equivalence $p_{1}+\pi_{\mathcal{P}}\left(p_{1}\right) \simeq p_{1}^{\prime}+\pi_{\mathcal{P}^{\prime}}\left(p_{1}^{\prime}\right)$. Finally, let $p$ be any other graph point contained in a representative of $p_{1}+\pi_{\mathcal{P}}\left(p_{1}\right)$. By Lemma 2.7, $\mathcal{P} \cup\left\{p_{1}^{\prime}, p\right\}$ is a rank determining set, so $p_{1}+\pi_{\mathcal{P}}\left(p_{1}\right)$ has rank 1 .

Now, suppose that $h=0,1$. Then by the construction in Proposition 2.6, there is a vertex $v_{0}$ such that the restriction $P_{0}$ of $\mathcal{P}$ to $C_{v_{0}}$ is rigid and of degree $g_{v_{0}}-1$. Find a point $p_{0}$ so that $P_{0}+p_{0}$ is rigid, and a graph point $p$ that is contained in a representative of $p_{1}+\pi_{\mathcal{P}}\left(p_{1}\right)$. Similarly to the higher genus case, $\mathcal{P} \cup\left\{p_{0}\right.$, $p$ \} is rank determining, and the divisor $p_{1}+\pi_{\mathcal{P}}\left(p_{1}\right)$ has representatives through each of the points in its support. We conclude that it has rank 1, and the proof is complete.

3.2. The structure of hyperelliptic metrized complexes. To complete our discussion on metrized complexes, we show that being hyperelliptic imposes strong conditions on their structure. The following characterization is familiar to experts, but to the extent of our knowledge, does not appear in the literature. For a visual illustration of the lemma, see Figure 1.

LEMMA 3.5. A metrized complex $\mathfrak{C}$ is hyperelliptic if and only if it satisfies the following properties: 
(1) The underlying graph $\Gamma$ is either a tree or hyperelliptic with involution $\iota_{\Gamma}$ (if $\Gamma$ is a tree then $\iota_{\Gamma}$ is just the identity).

(2) If $C_{v}$ has genus $g_{v}>0$, then $\iota_{\Gamma}(v)=v$. For every node $p$ corresponding to an edge e, the edge $\iota_{\Gamma}(e)$ meets $C_{v}$ at a node $p^{\prime}$, and all such divisors $p+p^{\prime}$ are equivalent.

(3) If $g_{v} \geqslant 2$, then $C_{v}$ is hyperelliptic with involution $\iota_{v}$, satisfying $\iota_{v}(p)=p^{\prime}$ for every node $p$.

Proof. Suppose that $\mathfrak{C}$ is hyperelliptic, and let $x+x^{\prime}$ be a divisor of degree 2 and rank 1 . When passing to the underlying graph, rank may only increase, so $x+x^{\prime}$ has rank at least 1 on $\Gamma$. By [9, Theorem 1.3], $\Gamma$ is either a tree or a hyperelliptic graph with involution $\iota_{\Gamma}$. Let $v$ be a vertex of $\Gamma$. By Lemma 2.4, there is a divisor equivalent to $x+x^{\prime}$ whose restriction to $C_{v}$ has rank at least 1 . When $g_{v}>0$, it implies that this restriction has degree 2. In particular, $v$ is a point of $\Gamma$ with $\iota_{\Gamma}(v)=v$. When $g_{v}>1$, we conclude that $C_{v}$ is hyperelliptic with involution $\iota_{v}$. Let $p, p^{\prime}$ be a pair of nodes on $C_{v}$ corresponding to edges $e^{\prime}=\iota_{\Gamma}(e)$. Then for every pair of points $y, y^{\prime}$ on $e, e^{\prime}$ at equal distance $\epsilon$ from $v, x+x^{\prime} \simeq y+y^{\prime}$. By letting $\epsilon$ tend to zero, we see that $x+x^{\prime} \simeq p+p^{\prime}$.

Conversely, suppose that the conditions above are satisfied. Let $x$ be a graph point of $\Gamma$, and let $x^{\prime}=\iota_{\Gamma}(x)$. For any other point $p$ of $\mathfrak{C}$, it can be verified, using Dhar's burning algorithm, that the $p$-reduced divisor equivalent to $p$ has a chip at $p$. In particular, the rank of $x+x^{\prime}$ is 1 .

We define a map $\iota$ on $\mathfrak{C}$ as follows. If $p$ is a point of $\mathfrak{C}$ that does not lie on a rational component, then

$$
\iota(p)= \begin{cases}\iota_{\Gamma}(p) & p \text { is a graph point of } \Gamma, \\ q & p \text { is a point of } C_{v} \text { with } g_{v}=1, \text { and } p+q \\ & \text { is equivalent to a pair of nodes on } C_{v}, \\ \iota_{v}(p) \quad & p \text { is a point of } C_{v} \text { with } g_{v} \geqslant 2 .\end{cases}
$$

If $p$ is on a rational component $C_{v}$, and $\iota_{\Gamma}(v)=v$, then define $\iota(p)=p$. Otherwise, $\iota(p)$ is any point of $C_{\iota(v)}$. As all the points on rational components are linearly equivalent, it does not matter, for purposes of divisor theory, which point we choose. For every $p \in \mathfrak{C}$, the rank of $p+\iota(p)$ is 1 . Let $\mathfrak{g}_{2}^{1}$ be the divisor class of $p+\iota(p)$ for some $p$.

Reduced divisors. Recall that a divisor $\mathcal{D}$ on a metrized complex is said to be $v$-reduced with respect to a point $v$ of $\Gamma$ if it is effective away from $v$, and its 
chips are as 'lexicographically close' as possible to $v$ (see [1, Section 3.1] for a precise definition). For any $v$, reduced divisors exist and are quasiunique, which means that their graph part is unique, and the restrictions to the components are unique up to linear equivalence [1, Theorem 3.7]. We extend the definition to nongraphical points of a metrized complex. A divisor $\mathcal{D}$ is $p$-reduced for a point $p$ on a component $C_{v}$, if it is $v$-reduced, and its restriction to $C_{v}$ has the highest degree at $p$ among the divisors that are effective away from $p$. Reduced divisors are quasiunique, and their restriction to $C_{v}$ is unique.

In [15], it was shown that every divisor of degree $d$ and rank $r$ (for $0 \leqslant r \leqslant$ $d \leqslant g$ ) on a hyperelliptic metric graphs contains $r$ copies of the unique divisor of degree 2 and rank 1. A similar argument holds for metrized complexes.

THEOREM 3.6. Let $\mathfrak{C}$ be a hyperelliptic metrized complex. Then every divisor class $\delta$ of degree $d$ and rank $r$ with $0 \leqslant r \leqslant d \leqslant g$ is equivalent to $r \cdot \mathfrak{g}_{2}^{1}+p_{2 r+1}+$ $\cdots+p_{d}$.

Proof. Let $p$ be a point of $\mathfrak{C}$ with $\iota(p)=p$. Let $\mathcal{D}$ be the $p$-reduced representative of $\delta$. It suffices to show that $\mathcal{D}(p) \geqslant 2 r$. First, assume that $r=1$. In this case, $\mathcal{D}(p) \geqslant 1$. Assume for the sake of contradiction that it equals 1 . Since $d \leqslant g$, it is follows from [1, Lemma 3.11] that there is a point $q \neq p$ of $\mathfrak{C}$ such that $\mathcal{D}+q$ is still $p$-reduced. Let $\mathcal{D}^{\prime}$ be the $p$-reduced representative of $\delta+p-\iota(q)$, and set $\mathcal{D}^{\prime \prime}=\mathcal{D}+q-p$. Then $\mathcal{D}^{\prime \prime} \simeq \mathcal{D}^{\prime}$, and both are $p$-reduced, because $p$-reducedness does not change when adding or subtracting chips at $p$. By quasiuniqueness, both divisors have the same number of chips at $p$. But $\mathcal{D}^{\prime \prime}(p)=0$ and $\mathcal{D}^{\prime}(p)$ is at least 1 , so we arrive at a contradiction.

For $r>1$, let $s$ be the largest integer so that $\mathcal{D}=2 s \cdot p+q_{1}+\cdots+q_{k}$ (where one of the points $q_{i}$ might coincide with $p$ ). We need to show that $s \geqslant r$. Again, by [1, Lemma 3.11], there are points $q_{k+1}, \ldots, q_{k+s}$ different from $p$, such that $\mathcal{D}+q_{k+1}+\cdots+q_{k+s}$ is $p$-reduced. Since $2 p$ is equivalent to $q+\iota(q)$ for every $q$, we have $\mathcal{D} \simeq q_{1}+\cdots+q_{k}+q_{k+1}+\iota\left(q_{k+1}\right)+\cdots+q_{k+s}+\iota\left(q_{k+s}\right)$. Therefore, the $p$-reduced representative of $\mathcal{D}-\iota\left(q_{k+1}\right)-\cdots-\iota\left(q_{k+s}\right)$ is exactly $q_{1}+\cdots+q_{k}$. Its degree at $p$ is at most 1 , so by the first part, its rank is 0 . This divisor was obtained from $\mathcal{D}$ by removing $s$ chips, so the rank of $\mathcal{D}$ is at most $s$.

\section{Acknowledgements}

I started thinking about this problem some time ago, following a meeting of the Yale lunch seminar, and I thank all the participants for the fruitful discussion. I am grateful to Dave Jensen, Dhruv Ranganathan, Sam Payne and Nick Wawrykow for insightful remarks on a previous version of this paper, and for pointing out a 
gap in the proof of Proposition 3.3. In addition, I had enlightening conversations with Spencer Backman, Marc Coppens, Ohad Feldheim and Jifeng Shen. Finally, I thank the nameless referees for their insightful suggestions and remarks. While writing this paper, I was supported by DFG grant MA 4797/1-2.

\section{Appendix A. Martens' theorem (joint with David Jensen)}

In this section, we discuss possibilities for a tropical version of Martens' theorem, which refines the characterization of hyperelliptic curves provided by Clifford. It is one of several structure theorems for classifying special curves according to their Brill-Noether loci. A further refinement is given by Mumford [4, Theorem 5.2], and on the other extreme, the Brill-Noether theorem determines the dimension of the Brill-Noether locus of general curves $[12,16,17]$. Let us first recall the classical statement of Martens' theorem.

THEOREM [4, Theorem 5.1]. Let $C$ be a smooth curve of genus $g$, and let $d$ and $r$ be integers satisfying $0<2 r \leqslant d<g$. Then $\operatorname{dim}\left(W_{d}^{r}(C)\right) \leqslant d-2 r$, and equality holds precisely when $C$ is hyperelliptic.

Note that the special case where $d=2 r$ is Clifford's theorem. As a first attempt, we examine a naive tropical analog of the theorem. As we see, the first part of the statement holds, but the second, unfortunately, does not.

Recall that the Jacobian of a metric graph $\Gamma$ has the structure of a real torus $\mathbb{R}^{g} / \mathbb{Z}^{g}$, where addition in the torus corresponds to addition of divisors [6, Theorem 3.4]. After fixing a base point $p_{0}$, the set of divisor classes of any degree $d$ can be identified with the Jacobian, by sending a divisor $D$ to $D-d \cdot p_{0}$. For instance, the set $W_{1}(\Gamma)$ is the image of the Abel-Jacobi map which sends every point $x$ of $\Gamma$ to $x-p_{0}$ in the Jacobian. Addition of divisors is still respected under this identification. Consequently, the set $W_{d}(\Gamma)$ of effective divisor classes of degree $d$ can be identified with the Minkowski sum of $d$ copies of $W_{1}(\Gamma)$. Moreover, since any divisor of degree $g$ is effective by Riemann-Roch, the Minkowski sum of $g$ copies of $W_{1}(\Gamma)$ is the entire Jacobian. In particular, $W_{1}(\Gamma)$ is nondegenerate, namely, it is not contained in any hyperplane quotient in $\mathbb{R}^{g} / \mathbb{Z}^{g}$.

THEOREM A.1. Let $\Gamma$ be a metric graph of genus $g$, and let $d$ and $r$ be integers satisfying $0<2 r \leqslant d<g$. The dimension of $W_{d}^{r}(\Gamma)$ is at most $d-2 r$, and equality holds for hyperelliptic graphs. However, the dimension of $W_{d}^{r}(\Gamma)$ may be $d-2 r$ for nonhyperelliptic curves. 
Proof. We prove the first part by induction on $r$. Let $r=1$. We need to show that $\operatorname{dim}\left(W_{d}^{1}(\Gamma)\right)<d-1$. The Brill-Noether locus in this case coincides with the set of divisor classes with an effective representative through every point of $\Gamma$. That is,

$$
W_{d}^{1}(\Gamma)=\bigcap_{p \in \Gamma}\left([p]+W_{d-1}(\Gamma)\right)
$$

Here, $[p]+W_{d-1}(\Gamma)$ is the translation in the Jacobian of $W_{d-1}(\Gamma)$ by the point representing the divisor class $[p]$. Since $[p]$ varies over all the points of the nondegenerate complex $W_{1}(\Gamma)$, and the dimension of $W_{d-1}(\Gamma)$ is smaller than $g$, Lemma A.2 below implies that the dimension of $W_{d}^{1}(\Gamma)$ is at most $d-2$, as claimed. Next, assume that the statement holds for $r-1$. We have

$$
W_{d}^{r}(\Gamma)=\bigcap_{p \in \Gamma}[p]+W_{d-1}^{r-1}(\Gamma) .
$$

By induction, all the cells of $W_{d-1}^{r-1}(\Gamma)$ are of dimension at most $d-2 r+1$, so Lemma A.2 implies that the intersection has dimension at most $d-2 r$.

Now, suppose that $\Gamma$ is hyperelliptic, and let $\delta$ be an element of $W_{d}^{r}(\Gamma)$. By Proposition 3.6, $\delta$ has a representative of the form $w_{1}+\cdots+w_{d-2 r}+2 r \cdot v_{0}$, where $v_{0}$ is a point of $\Gamma$ which is invariant under the hyperelliptic involution. Since $2 r \cdot v_{0}$ has rank $r$, any perturbation of the points $w_{1}, \ldots, w_{d-2 r}$ results in a divisor of rank at least $r$. It follows that $\delta$ has a neighborhood of dimension $d-2 r$ in $W_{d}^{r}(\Gamma)$.

To show that the converse is false, let $\Gamma$ be the graph from [19, Theorem 1.2]. It is straightforward to check that $\Gamma$ is not hyperelliptic. However, the dimension of $W_{3}^{1}(\Gamma)$ is $1=3-2 \cdot 1$.

LEMMA A.2. Let $\Sigma$ be a polyhedral complex of dimension $d<g$ in $\mathbb{R}^{g} / \mathbb{Z}^{g}$, and let $\Gamma$ be a nondegenerate 1-dimensional complex. Then for every cell $\sigma$ of $\Sigma$, there exists $p$ in $\Gamma$ such that the intersection of $p+\Sigma$ and $\sigma$ has dimension strictly smaller than $d$.

Proof. Let $\sigma$ be a $d$-dimensional cell $\Sigma$. Since $\Gamma$ is nondegenerate, it has an edge $e$ which is not contained in any translation of the affine subspace spanned by $\sigma$. Let $\sigma^{\prime}$ be another maximal cell of $\Sigma$. Then there is at most a single point $q$ in $e$ such that $\sigma^{\prime}+q$ and $\sigma$ intersect at dimension $d$. By varying over all possible $\sigma^{\prime}$, we see that there is a point $p$ in $e$ such that every cell in $\Gamma+p$ intersects $\sigma$ at positive codimension.

The theorem above is not the first example in which the dimension of the BrillNoether locus exhibits unpleasing behavior. For instance, it does not vary upper 
semicontinuously on the moduli space of tropical curves [19, Theorem 1.2]. These phenomena suggest that a different quantity should act as the tropical analog for the dimension of the Brill-Noether locus. Such an invariant was introduced in [19].

DEFINITION A.3. Let $X$ be either an algebraic curve, a metrized complex or a metric graph. Its Brill-Noether rank, denoted $w_{d}^{r}(X)$ is the largest number $\rho$ such that every effective divisor of degree $r+\rho$ is contained in a divisor of degree $d$ and rank $r$.

For an algebraic curve, the Brill-Noether rank coincides with the dimension of the largest component of its Brill-Noether locus. Consequently, it satisfies a specialization lemma: if $\Gamma$ is the skeleton of an algebraic curve $C$, and $\mathfrak{C}$ is the corresponding metrized complex, then $\operatorname{dim}\left(W_{d}^{r}(C)\right) \leqslant w_{d}^{r}(\mathfrak{C}) \leqslant w_{d}^{r}(\Gamma)$. Furthermore, it varies upper semicontinuously on the moduli space of tropical curves ([18, Theorems 5.1, 5.3] and [19, Theorems 1.6, 1.7]). As we show here, it also satisfies the first part of Martens' theorem.

Proposition A.4. Let $\mathfrak{C}$ be a metrized complex of genus $g$, and let $r, d$ be as in the conditions of Martens' theorem. Then

$$
w_{d}^{r}(\mathfrak{C}) \leqslant d-2 r .
$$

Moreover, for hyperelliptic complexes, $w_{d}^{r}(\mathfrak{C})=d-2 r$.

Proof. Suppose for contradiction that $w_{d}^{r}>d-2 r$. Then every divisor of degree $d-r+1$ is contained in a divisor of degree $d$ and rank $r$. But this is clearly false: choose a divisor of degree $d-r+1$ and rank 0 . By adding $r-1$ chips to any divisor, the rank may increase by at most $r-1$.

For the second part, assume that $\mathfrak{C}$ is hyperelliptic, and let $\mathcal{E}$ be an effective divisor of degree $d-r$. We need to show that it is contained in a divisor of degree $d$ and rank at least $r$. Since $d \geqslant 2 r$, the degree of $\mathcal{E}$ is at least $r$. Let $p_{1}, \ldots$, $p_{r}$ be points in the support of $\mathcal{E}$, and let $\iota$ be the hyperelliptic involution. Then $\mathcal{E}+\iota\left(p_{1}\right)+\cdots+\iota\left(p_{r}\right)$ has rank at least $r$.

Given the facts above, we speculate that Martens' theorem holds in tropical geometry.

Conjecture. Let $\mathfrak{C}$ be a metric graph of genus $g$, and let $d, r$ be such that $0<2 r \leqslant d<g$. Then $w_{d}^{r}(\mathfrak{C}) \leqslant d-2 r$, and equality holds precisely exactly when $\mathfrak{C}$ is hyperelliptic.

Very little is known about the conjecture, even in the metric graph case. 


\section{References}

[1] O. Amini and M. Baker, 'Linear series on metrized complexes of algebraic curves', Math. Ann. 362(1-2) (2015), 55-106.

[2] O. Amini, M. Baker, E. Brugallé and J. Rabinoff, 'Lifting harmonic morphisms II: tropical curves and metrized complexes', Algebra Number Theory 9(2) (2015), 267-315.

[3] O. Amini and L. Caporaso, 'Riemann-Roch theory for weighted graphs and tropical curves', Adv. Math. 240 (2013), 1-23.

[4] E. Arbarello, M. Cornalba, P. A. Griffiths and J. Harris, Geometry of Algebraic Curves, Vol. I, Grundlehren der Mathematischen Wissenschaften [Fundamental Principles of Mathematical Sciences, 267] (Springer, New York, 1985).

[5] M. Baker, 'Specialization of linear systems from curves to graphs', Algebra Number Theory 2(6) (2008), 613-653.

[6] M. Baker and X. Faber, 'Metric properties of the tropical Abel-Jacobi map', J. Algebraic Combin. 33(3) (2011), 349-381.

[7] M. Baker and D. Jensen, Degeneration of Linear Series from the Tropical Point of View and Applications (Springer, Cham, 2016), 365-433.

[8] M. Baker and S. Norine, 'Riemann-Roch and Abel-Jacobi theory on a finite graph', Adv. Math. 215(2) (2007), 766-788.

[9] M. Chan, 'Tropical hyperelliptic curves', J. Algebraic Combin. 37(2) (2013), 331-359.

[10] M. Coppens, 'Clifford's theorem for graphs', Preprint, 2013, arXiv:1304.6101.

[11] A. Gathmann and M. Kerber, 'A Riemann-Roch theorem in tropical geometry', Math. Z. 259(1) (2008), 217-230.

[12] P. Griffiths and J. Harris, 'On the variety of special linear systems on a general algebraic curve', Duke Math. J. 47(1) (1980), 233-272.

[13] C. Haase, G. Musiker and J. Yu, 'Linear systems on tropical curves', Math. Z. 270(3-4) (2012), 1111-1140.

[14] E. Katz, J. Rabinoff and D. Zureick-Brown, 'Uniform bounds for the number of rational points on curves of small mordell-weil rank', Duke Math. J. 165(16) (2016), 3189-3240.

[15] S. Kawaguchi and K. Yamaki, 'Rank of divisors on hyperelliptic curves and graphs under specialization', Int. Math. Res. Not. IMRN 2015(12) (2015), 4121-4176.

[16] G. Kempf, 'Schubert methods with an application to algebraic curves', Publ. Math. Centrum (1971).

[17] L. Kleiman and D. Laksov, 'On the existence of special divisors', Amer. J. Math. 94(2) (1972), 431-436.

[18] Y. Len, 'The Brill-Noether rank of a tropical curve', J. Algebraic Combin. 40(3) (2014), 841-860.

[19] C. M. Lim, S. Payne and N. Potashnik, 'A note on Brill-Noether theory and rank-determining sets for metric graphs', Int. Math. Res. Not. IMRN 2012(23) (2012), 5484-5504.

[20] G. Mikhalkin and I. Zharkov, 'Tropical curves, their Jacobians and theta functions', in Curves and Abelian Varieties, Contemporary Mathematics, 465 (American Mathematical Society, Providence, RI, 2008), 203-230. 